\title{
Light Emitting Diode Fluorescence Microscopy increased the detection of smear-positives during follow-up of Tuberculosis patients in India: program implications
}

\author{
Badri Thapa*, Lord Wasim Reza, Ajay MV Kumar, Ashish Pandey, Srinath Satyanarayana and Sarabjit Chadha
}

\begin{abstract}
Background: In India, since July 2012, at designated Microscopy Centers (DMCs) in 200 medical colleges, sputum smear examination for tuberculosis bacilli changed from Ziehl Neelsen (ZN) method to auramine based Light Emitting Diode Fluorescent Microscopy (LED-FM) method. We assessed the additional yield of smear positives among patients undergoing follow-up sputum examination during TB treatment before and after deploying LED-FM.

Methods: This was a before and after comparison study in eight conveniently selected medical college DMCs across North India. We extracted data from TB laboratory registers on number of TB patients examined for follow-up and their smear microscopy results including the grades by ZN (before; July-December 2011) and LED-FM (after; JulyDecember 2012) and compared them.

Results: Altogether, 2868 TB patients were examined by LED-FM and 2740 were examined by ZN during follow-up. LED-FM increased the proportion of follow-up smear positives from $5.0 \%(n=136)$ to $7.4 \%(n=213)$ with an additional yield of 77 follow-up smear-positives - with the highest increase in smears graded scanty (2.6 vs $1.2 \%$ ) ( $p$ value $<0.05)$.

Conclusions: Since all smear positives during follow-up are considered 'presumptive multidrug resistant (MDR)-TB patients' in India, introduction of LED-FM would result in additional number of patients eligible for MDR-TB testing, which would have otherwise been missed by ZN.
\end{abstract}

Keywords: LED-fluorescence microscopy, Follow-up smears, TB patients

\section{Background}

The World Health Organization (WHO) recommends phasing-in Light Emitting Diode Fluorescent Microscopy (LED-FM) as an alternative to conventional Ziehl-Neelsen microscopy (ZN) for diagnosis of smearpositive pulmonary tuberculosis (TB) [1]. Many studies

\footnotetext{
*Correspondence: badri_bishal@yahoo.com

Tuberculosis Department, International Union Against Tuberculosis

and Lung Disease, South-East Asia Regional Office, C-6, Qutub

Institutional Area, 110016 New Delhi, India
}

in controlled laboratory settings have shown that LED$\mathrm{FM}$ is about $10 \%$ more sensitive and as specific as $\mathrm{ZN}$ in detecting TB bacillus on smear microscopy [2]. In India, since July 2012 under the Revised National Tuberculosis Control Programme, the sputum smear examination for TB bacilli at designated microscopy centers (DMCs) in 200 medical colleges, changed from ZN method to auramine based LED-FM method. LED-FM has been shown to increase the detection of smear positive tuberculosis under routine programmatic conditions [3].

Unlike at the time of diagnosis, TB patients who undergo follow-up sputum smear examination during 
TB treatment, produce less sputum and are mostly paucibacillary [4]. There is no evidence from India if use of LED-FM increases the detection of smear positives among patients undergoing follow-up sputum smear examination under routine programmatic conditions. In this study, we evaluated whether implementation of LEDFM increased the yield of smear positives during followup and discuss its programmatic implications.

\section{Methodology}

We conducted a before and after comparison study in eight conveniently selected medical colleges in North India that were implementing LED-FM services. The study sample included all TB patients who underwent follow-up sputum smear examination in these colleges during the period July-December 2012 (using LED-FM) and July-December 2011 (using ZN microscopy). TB patients, as a part of monitoring their response to treatment, undergo follow-up smear examination at the end of intensive phase, 2 months into continuation phase and at the end of treatment. Those found to be sputum smear positive are evaluated for drug resistance. Patient details, reasons for sputum smear examination (whether for diagnosis or for follow-up) and results are recorded in the TB laboratory register. For this study, we included all patients who under-went follow-up sputum smear examination conducted during the two study periods. We reviewed the TB laboratory registers and abstracted aggregate data on number of TB patients examined for follow-up and their smear microscopy results including grades.

LED-FM and ZN procedures were performed by trained laboratory technicians. As per programme guidelines, smears were prepared from direct sputum specimens in both techniques. Briefly, for LED-FM, the heat fixed smears were flooded with $0.1 \%$ auramine phenol for 7-10 min, rinsed with running water, decolorized with $3 \%$ acid alcohol for 2 min twice, rinsed with running water, counter stained with $0.1 \%$ potassium permanganate for $30 \mathrm{~s}$, rinsed with running water and air dried [4]. For ZN microscopy, the heat fixed smears were flooded with $1 \%$ carbol fuchsin for $5 \mathrm{~min}$, rinsed gently with running water, decolorized with $25 \%$ sulphuric acid for 2-4 min, rinsed with water, counter stained with $0.1 \%$ methylene blue for $30 \mathrm{~s}$, rinsed with running water and air dried [5]. Trained laboratory technicians prepared and examined the smears and reported the results as shown in Table 1 [3]. The microscopy centers performed smear microscopy procedures under a system of external quality assurance which included onsite visits by dedicated laboratory supervisors and periodic, random blinded rechecking of smears [6,7]. As per national guidelines, two smears are required to be examined for follow up examinations [6]. Patient will be labelled as smear positive at follow-up if at least one smear is positive irrespective of the grades. If both smears are positive, the higher microscopy grade is used for reporting. As per the newer national guidelines on programmatic management of drug resistant TB, all TB patients who are smear positive during follow-up at the end of 2 months of intensive phase and/or 5 months of continuous phase (depending upon the districts) are considered as 'presumptive multidrug resistant (MDR)-TB patients' and are investigated for Rifampicin and Isoniazid resistance using culture and drug susceptibility test (CDST) [8]. If patients are confirmed to be having strains that are resistant to Rifampicin in vitro, they are treated using WHOrecommended standardized second line regimen.

The number of TB patients examined during follow-up and proportion found smear positive was computed, separately for LED-FM and ZN microscopy. Chi squared test was used to test statistical significance ( $p$-value $\leq 0.05$ ) (SPSS v16). Ethics approval for this study was obtained from the Ethics Advisory Group of International Union Against Tuberculosis and Lung Disease (The Union).

\section{Results}

Of 2868 TB patients examined by LED-FM during follow-up, $213(7.4 \%)$ were smear-positive and of 2740 examined by ZN, 136 (5.0 \%) were smear-positive-an

Table 1 Guidelines of reporting sputum smear microscopy adopted by National Tuberculosis Control Programme in India

\begin{tabular}{|c|c|c|}
\hline $\begin{array}{l}\text { IUATLD/WHO scale }\left(1000 \times \text { field }=\text { HPF }^{*}\right) \\
\text { Grades }\end{array}$ & $\begin{array}{l}\text { Ziehl Neelsen (ZN) microscopy }(1000 \times \\
\text { magnification: } 1 \text { length }=2 \mathrm{~cm}=100 \mathrm{HPF})\end{array}$ & $\begin{array}{l}\text { LED-Fluorescence microscopy }(400 \times \\
\text { magnification: } 1 \text { length }=40 \text { fields }=200 \mathrm{HPF})\end{array}$ \\
\hline Negative & Zero AFB/1 length & Zero AFB/1 length \\
\hline \multicolumn{3}{|l|}{ Positive } \\
\hline Scanty & 1-9 AFB/1 length or $100 \mathrm{HPF}$ & 1-19 AFB/1 length or $100 \mathrm{HPF}$ \\
\hline $1+$ grade & 10-99 AFB/1 length or $100 \mathrm{HPF}$ & 20-199 AFB/1 length \\
\hline $2+$ grade & 1-10 AFB/1 HPF on average & 5-50 AFB/1 HPF on average \\
\hline $3+$ grade & $>10 \mathrm{AFB} / 1 \mathrm{HPF}$ on average & $>50 \mathrm{AFB} / 1 \mathrm{HPF}$ on average \\
\hline
\end{tabular}

* HPF high power field 
additional yield of 77 follow-up smear-positives. The disaggregated results of first and second specimens are shown in Table 2. The additional yield of second specimen (negative on first specimen and positive only on second specimen) was $12.5 \%$ with $\mathrm{ZN}$ microscopy whereas it was $21.6 \%$ with LED-FM ( $\mathrm{p}=0.3$ ).

Compared to the before period, in the after-period there was a $5 \%$ increase in the number of TB patients who underwent follow-up sputum smear examination. However, the number of TB patients found to be sputum smear positive rose by more than $50 \%$ (Table 2). Applying $5.0 \%$ positivity (observed in 2011) for the additional number of patients examined in 2012, LED-FM services still resulted in an additional yield of 71 follow-up smearpositives. Most of the increase was noted in smears graded scanty followed by $3+($ p-value $<0.05)$ (Table 3 ). The direct yield was higher by LED-FM in most $(n=7)$ sites compared.

\section{Discussion}

This is the first study from India examining the impact of LED-FM on the sputum-positivity during follow-up of TB patients under routine programmatic settings. The increase in yield by LED-FM could be due to (1) increased absorbability of mycolic acid and damaged bacilli to auramine than fuschin, (2) the LED-FM stained background of slide is brighter, making it easier for laboratory technicians to focus and examine slides with fewer bacilli and (3) increased area of the slide examined [7, 9]. Since these findings come from a setting of qualityassured smear microscopy under routine programmatic settings, they are most likely to be accurate and reflect operational realities and importantly during the period of study external quality assurance did not yield any major errors.

These results have important programme implications. LED-FM leads to an increase in the number of TB patients who will be identified as "presumptive MDR-TB patients" who will require CDST with consequent increase in laboratory workload. Several studies in the past have shown that nearly two-thirds of the smear positives during follow-up were culture negative, especially so if the smears are graded scanty, which most likely represent dead, nonviable bacilli, yet to be excreted out of the body $[10,11]$. Hence, it is not clear if increase in identification of followup smear-positives using LED-FM is actually beneficial in enhancing detection of MDR-TB cases or just unnecessary additional workload for the CDST laboratories. Since we do not have information on culture results in our study, the possibility of false positive results could not

Table 2 Performance of LED-FM (July-December 2012) in comparison to ZN microscopy (July-December 2011) in detecting AFB during follow-up of TB patients in eight medical colleges of India

\begin{tabular}{|c|c|c|c|c|c|c|c|}
\hline Technique & $\begin{array}{l}\text { Number } \\
\text { examined }\end{array}$ & $\begin{array}{l}\text { Number smear } \\
\text { positive }\end{array}$ & PP & PN & NP & $\begin{array}{l}\text { Proportion smear } \\
\text { positive }\end{array}$ & 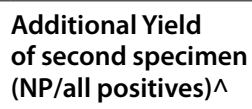 \\
\hline ZN & 2740 & 136 & 110 & 9 & 17 & $5.0 \%$ & $12.5 \%$ \\
\hline LED-FM & 2868 & 213 & 149 & 18 & 46 & $7.4 \%$ & $21.6 \%$ \\
\hline $\begin{array}{l}\text { Absolute increase (rela- } \\
\text { tive increase) }\end{array}$ & $+128(4.7 \%)$ & +77 (56.6 \%) & & & & $+2.4 \%(48 \%)$ & \\
\hline
\end{tabular}

ZN Ziehl Neelsen Microscopy, LED-FM light emitting diode fluorescence microscopy, AFB acid fast bacilli, $P P$ first and second smear positive, $P N$ first smear positive and second smear negative, NP first smear negative and second smear positive

\# $\mathrm{p}$ value $<0.001$

$\wedge p$ value $=0.03$

Table 3 Comparison between ZiehI Neelson (ZN) and Light Emitting Diode-Fluorescence Microscopic technique (LED-FM) in detecting AFB (by smear quantification) during follow-up of TB patients in eight medical colleges of India

\begin{tabular}{|c|c|c|c|c|c|c|c|}
\hline \multicolumn{2}{|c|}{ Smear grade of patient } & \multicolumn{2}{|c|}{ ZN Microscopy 2011} & \multicolumn{2}{|c|}{ LED-FM 2012} & \multirow[t]{2}{*}{ Change in percentage $(95 \% \mathrm{Cl})$} & \multirow[t]{2}{*}{ p-value } \\
\hline & & $\mathrm{n} 1$ & $\%$ & $\mathrm{n} 2$ & $\%$ & & \\
\hline Negative & & 2604 & 95.0 & 2655 & 92.6 & $-2.4(-1.1$ to -3.7$)$ & $<0.0001$ \\
\hline \multirow[t]{4}{*}{ Positive } & Scanty & 33 & 1.2 & 75 & 2.6 & $1.4(0.7-2.0)$ & 0.0001 \\
\hline & $1+$ & 57 & 2.1 & 78 & 2.7 & $0.6(-0.1$ to 1.4$)$ & 0.14 \\
\hline & $2+$ & 29 & 1.1 & 27 & 0.9 & $-0.2(-0.7$ to 0.4$)$ & 0.45 \\
\hline & $3+$ & 17 & 0.6 & 33 & 1.1 & 0.5 (0.0 to 1.0$)$ & 0.01 \\
\hline Total & & 2740 & 100.0 & 2868 & 100.0 & - & - \\
\hline
\end{tabular}

ZN ZiehI Neelsen Microscopy, LED-FM light emitting diode fluorescence microscopy, AFB acid fast bacilli 
be assessed and should be a topic for future studies. To avoid this 'smear-positive culture-negative' phenomenon and increase the specificity of smear microscopy during follow-up, The Union in their TB guidelines recommend that all follow-up smear-positives (especially that done during month 5) in the first sample be reconfirmed by a second positive smear using an early morning sputum sample before declaring as 'treatment failure' and start next steps including investigation for MDR-TB [11]. A recent study from Chennai showed that if only one of the two smears was positive, this is most likely to be scanty and hence culture negative [12]. Adopting the Union's approach would mean that TB patients who are positive on both the smears only would be investigated for MDR$\mathrm{TB}$ and would thus reduce unnecessary workload. The other alternatives to reduce unnecessary cultures are to use new methods like vital staining with fluorescein diacetate or slide culture, but these are not widely available or validated under routine conditions [13-15].

There were a few limitations to this study. It would have been better if the same samples were processed with the two methods. However, this could not be performed as the $\mathrm{ZN}$ microscopy system was already replaced with the LED-FM microscopy in the study sites. The culture and DST results were unavailable and hence it could not be found out if use of LED-FM actually increased the detection of MDR-TB cases. As in all pre-post designs, it is difficult to attribute the entire change to the intervention (LED-FM) as there could be other undocumented changes (for example, pre-treatment smear positivity) during the study period. While we do not suspect this, we have no data to rule it out completely and is one of the limitations. Also the study did not disaggregate the follow-up smears at end of intensive phase and end of treatment. Since the study was conducted in conveniently selected medical colleges, there may be difficulties in generalizing the results to whole of India. Further studies should collect data countrywide or from a representative sample to confirm these results and act upon them.

\section{Conclusions}

Implementation of LED-FM led to increase in identification of follow-up smear-positives among TB patients, mostly graded scanty. This has important resource implications for the TB programme in India.

\section{Authors' contributions}

BT designed the study, collected and analysed the data, drafted the manuscript, LWR designed the study and revised the manuscript, AMVK, AP, SS and $S C$ revised the manuscript. All authors read and approved the final manuscript. or interpretation of data. The donor had no role in study design, data collection and analysis, decision to publish or preparation of the manuscript. The contents of this paper exclusively reflect the view of authors.

\section{Competing interests}

The authors declare that they have no competing interests.

Received: 28 November 2014 Accepted: 14 October 2015

Published online: 23 October 2015

\section{References}

1. World Health Organization. Fluorescent light emitting diode (LED) microscopy for diagnosis of tuberculosis: policy statement. Geneva: World Health Organization; 2010.

2. Steingart KR, Henry M, Ng V, Hopewell PC, Ramsay A, Cunningham J, et al. Fluorescence versus conventional sputum smear microscopy for tuberculosis: a systematic review. Lancet Infect Dis. 2006;6:570-81.

3. Reza LW, Satyanarayana S, Pandey A, Kumar S, Pandey A, Devendrappa $\mathrm{NM}$, et al. LED fluorescence microscopy increases the detection of smearpositive pulmonary tuberculosis in medical colleges of India. Public Health Action. 2013;3:240-2.

4. Nataraj G, Kanade S, Parikh R, Khatri V, Mehta P, Athavale A, et al. Incremental yield in sputum smear positivity by examining a second early morning sputum specimen in follow-up patients on DOTS: 7 year analysis of RNTCP laboratory register. Indian J Tuberc. 2011;58:60-5.

5. Central Tuberculosis Division: Module for laboratory technician. New Delhi: Directorate General of Health Services, Ministry of Health and Family Welfare, Government of India; 2005.

6. Central Tuberculosis Division: Manual for sputum smear fluorescence microscopy. New Delhi: Directorate General of Health Services, Ministry of Health and Family Welfare, Government of India; 2011.

7. Central Tuberculosis Division: Revised National Tuberculosis Control Program Technical and operational guidelines for tuberculosis control. New Delhi: Directorate General of Health Services, Ministry of Health and Family Welfare, Government of India; 2005.

8. Xia H, Song YY, Zhao B, Kam KM, O'Brien RJ, Zhang ZY, et al. Multicentre evaluation of Ziehl-Neelsen and light-emitting diode fluorescence microscopy in China. Int J Tuberc Lung Dis. 2013;17:107-12.

9. Central Tuberculosis Division: Guideline on programmatic management of drug resistant TB (PMDT) in India. Revised National Tuberculosis Control Program: Directorate General of Health Services, Ministry of Health and Family Welfare, Government of India; 2012.

10. Reza LW, Satyanarayna S, Enarson DA, Kumar AM, Sagili K, Kumar S, et al. LED-fluorescence microscopy for diagnosis of pulmonary tuberculosis under programmatic conditions in India. PLoS One. 2013;9(8):e75566. doi:10.1371/journal.pone.0075566 (eCollection 2013).

11. Chamie G, Charlebois ED, Srikantiah P, Walusimbi-Nanteza M, Mugerwa RD, Mayania H, et al. Mycobacterium tuberculosis microbiologic and clinical treatment outcomes in a randomized trial of immediate versus $\mathrm{CD}^{+}{ }^{-}$-initiated antiretroviral therapy in HIV-infected adults with a high CD4 $^{+}$cell count. Clin Infect Dis. 2010;51:359-62.

12. Enarson DA, Reider HL, Arnadottir, Trebucq A. Management of tuberculosis. 5th ed. France: International Union Against Tuberculosis Control and Lung Disease; 2000.

13. Kumar RS, Kumar AMV, Claassens M, Banurekha W, Gomathi NS, Venkatesan $\mathrm{P}$, et al. Number of sputum specimens during treatment follow-up of Tuberculosis patients-two or one? Pub Health Act. 2013;3:304-7.

14. Van Deun A, Maug AKJ, Hossain A, Gumusboga M, de Jong BC. Fluorescein diacetate vital staining allows earlier diagnosis of rifampicin-resistant tuberculosis. Int J Tuberc Lung Dis. 2012;16:1174-9.

15. Hemavathi SP, Ramesh DH. Comparative evaluation of the rapid slide culture and microscopy with the conventional culture method in the diagnosis of pulmonary tuberculosis. J Clin Diag Res. 2012;6:192-4.

\section{Acknowledgements}

This project was supported by TB Reach Initiative of Stop TB Partnership (through a grant from the Canadian International Development Agency) grant no T9-370-114. Funders had no role in study design, data collection, analysis 